\title{
Quantifying exchange coupling in $f$-ion pairs using the diamagnetic substitution method
}

\author{
Wayne W. Lukens*1 and Marc D. Walter ${ }^{2}$
}

1) Actinide Chemistry Group, Chemical Sciences Division, Lawrence Berkeley National Laboratory, Berkeley, California 97420

2) Department of Chemistry, University of California, Berkeley, California 94720

wwlukens@lbl.gov

RECEIVED DATE (to be automatically inserted after your manuscript is accepted if required according to the journal that you are submitting your paper to)

* Email: wwlukens@lbl.gov

\begin{abstract}
One of the challenges in the chemistry of actinide and lanthanide ( $f$-ion) is quantifying exchange coupling between $f$-ions. While qualitative information about exchange coupling may be readily obtained using the diamagnetic substitution approach, obtaining quantitative information is much more difficult. This article describes how exchange coupling may be quantified using the susceptibility of a magnetically isolated analog, as in the diamagnetic substitution approach,
\end{abstract}


along with the anisotropy of the ground state as determined by EPR spectroscopy. Several examples are used to illustrate and test this approach.

Keywords (magnetic susceptibility, exchange coupling, actinide, lanthanide, anisotropic coupling, diamagnetic substitution, cerium, uranium, ytterbium)

\section{Introduction}

One major challenge in the chemistry and physics of actinide and lanthanide ( $f$-ion) complexes is understanding the role that $f$-electrons play in magnetism in general and in exchange-coupling in particular. ${ }^{1,2}$ This is in marked contrast to transition metal magnetochemistry where a straightforward spin-only Hamiltonian approach can clarify magnetic behavior and quantify the exchange coupling of $d$-electrons..$^{3-5}$ Other than $f^{3}$-systems, exchange coupling in few actinide and lanthanide complexes has been analyzed quantitatively since a spin-only Hamiltonian is not applicable to systems with unquenched orbital angular momenta. This difficulty complicates efforts to explain the interesting and fundamentally important magnetic behavior of lanthanide and actinide complexes. Some recent examples include work by Booth and Walter et al., which has shown that the behavior of certain $\mathrm{Ce}$ and $\mathrm{Yb}$ complexes can only be rationalized within a framework of multiconfigurational, Kondo-like, ground states. ${ }^{6-8}$ In addition, Schelter, et al. have shown that intramolecular coupling exists between $\mathrm{U}$ and $\mathrm{Yb}$ centers in polymetallic clusters, ${ }^{9}$ and Ishikawa et al. have demonstrated that phthalocyanine complexes of the lanthanides possess interesting magnetic properties. ${ }^{10-13}$ In addition, a large number of studies have examined the coupling between $f$-ions and adjacent paramagnetic transition metals or organic radicals..$^{1,14-27}$ 
Two factors make it challenging to quantify $f$-electron coupling. First, the magnetic moments of $f$-ions, except for $f^{\prime}$-systems, are temperature dependent and highly anisotropic due to a combination of strong spin-orbit coupling and weak crystal fields. ${ }^{18}$ The crystal field splits each ${ }^{2 \mathrm{~S}+1} \mathrm{~L}_{\eta}$ free-ion term, where $\mathrm{S}$ is the spin, $\mathrm{L}$ is the orbital angular momentum, and $g$ is the total angular momentum of the term, into series of states (Stark sublevels), where the total splitting varies from approximately $10^{2}$ to $10^{3} \mathrm{~cm}^{-1} \cdot{ }^{28}$ Consequently, the populations of the low-lying states change over the temperature range of susceptibility experiments, which renders the effective magnetic moments of these complexes highly temperature dependent. The second factor is the fact that the exchange interaction between adjacent $f$-metal centers is highly anisotropic, largely due to anisotropy in the spectroscopic splitting factor, $\mathbf{g} .^{28}$ These two factors, temperature dependent moments and anisotropic coupling, generally preclude the use of the simple spin-only Hamiltonian, and require other approaches to investigate exchange-coupling between $f$-ions.

Ideally, exchange coupling may be investigated directly by diagonalizing a Hamiltonian that includes contributions from free-ion, crystal field, Zeeman, and exchange-coupling terms: $\mathrm{H}_{\mathrm{tot}}=\mathrm{H}_{\mathrm{FI}}+\mathrm{H}_{\mathrm{CF}}+\mathrm{H}_{\mathrm{Ze}}+\mathrm{H}_{\mathrm{Exch}}$. This approach is technically challenging but has been successfully applied to exchange coupled systems involving $f$-ions..$^{11,18,24,25,27,29,30}$ The great advantage of this approach is that it can provide a detailed understanding of exchange coupling in $f$-ion systems as it explicitly describes the wave functions of the states of the $f$-ion and the paramagnetic moiety to which it is coupled. The biggest challenge to implementing this approach is that it requires accurate crystal field parameters, which can be very difficult to determine. For complexes 
involving organic ligands, accurate crystal field parameters are known for only a few, highly symmetric systems. Nevertheless, if accurate crystal field parameters can be determined, this approach provides the most detailed description of exchange coupling.

Due to the difficulty in determining crystal field parameters accurately, especially when the complexes are not highly symmetric, the preceding approach is not widely employed for $f$-ions in organic complexes (as opposed to $f$-ions in inorganic matrices), so other methods are typically used to investigate exchange coupling in such systems. If the excited states are significantly higher in energy than the ground state and the degree of anisotropy of the coupling between the $f$-ions is known, the susceptibility can be analyzed using a spin-only Hamiltonian. This approach was used by Rosen, et al. to model the susceptibility of a pentavalent uranium complex with a bridging imido ligand, $\left[(\mathrm{MeCp})_{3} \mathrm{U}\right]_{2}\left(\mu-1,4-\mathrm{N}_{2} \mathrm{C}_{6} \mathrm{H}_{4}\right)$, where $\mathrm{MeCp}$ is methylcyclopentadienyl, and 1,4- $\mathrm{N}_{2} \mathrm{C}_{6} \mathrm{H}_{4}$ is 1 ,4-benzenediimide. ${ }^{31}$ Recently, the same method has been applied to another pentavalent uranium complex consisting of a pair of uranium bis-imido moieties. ${ }^{32}$ It should be noted that both systems involve $\mathrm{U}(\mathrm{V})$ multiply-bonded to imide ligands, which results in the strong crystal field required for this approach. While this approach is straightforward, the requirement for a well-isolated ground state limits the instances where it may be applied.

The most widely used approach to investigate coupling between f-ions with unknown crystal field parameters is the diamagnetic substitution method, in which the susceptibility of an exchange-coupled pair of $f$-ions is evaluated by comparing it to the susceptibility of a magnetically isolated analog, which is usually prepared by replacing one $f$-ion by a diamagnetic equivalent, such as $\mathrm{Lu}$ or $\mathrm{La} .{ }^{15,16,33}$ The magnetically isolated analog has a very similar crystal 
field to the original $f$-ion pair but lacks the complicating effect of the coupling between the $\mathrm{f}$ ions. The susceptibility of the magnetically isolated analog may be subtracted from the susceptibility of the coupled system to determine whether the coupling is ferromagnetic or antiferromagnetic. Recent work by Long and coworkers has extended this approach to provide information about the coupling of excited states in uranium complexes. ${ }^{26,34}$

As noted above, the diamagnetic substitution method is widely used to determine qualitative information about coupling between f-ions, namely whether the coupling is ferromagnetic or antiferromagnetic. The principal reason that this approach is so widely used is that it allows the information about the crystal field contained in the susceptibility of the magnetically isolated analog to be used to analyze the coupling between f-ions without having to determine the crystal field parameters. This manuscript describes how this information, along with the magnetic anisotropy of ground state, typically determined by EPR spectroscopy, can be used to determine the phenomenological coupling between both the effective spins of the $f$-ions and the true spins of the $f$-electrons.

\section{Experimental}

Data for the lanthanide phthalocyanine triple-decker complexes were scanned and digitized from ref 11. Data for the cerium, ytterbium, and uranium complexes were provided by the authors of refs $7,35,36$, and 31 . All susceptibility data analysis, including least-squares fitting, was performed using Microsoft Excel. $\mathrm{Cp}_{2}{ }_{2} \mathrm{Yb}(\mathrm{bipy}) \mathrm{I}$ was prepared as previously reported. ${ }^{36}$ Electron paramagnetic resonance (EPR) spectra were obtained at $1.5 \mathrm{~K}$ using a Varian E-12 spectrometer equipped with an EIP-547 microwave frequency counter and a Varian E-500 
gaussmeter that was calibrated using 2,2-diphenyl-1-picrylhydrazyl. The EPR spectrum was fit using a version of the code ABVG modified to allow anisotropic line-widths and to fit spectra using the downhill simplex method. ${ }^{37}$

\section{Results and Discussion}

\section{Quantitative diamagnetic substitution approach}

The logic used to develop the theoretical model closely follows that outlined by Lines to describe the coupling between two high-spin $\mathrm{Co}$ (II) ions; however, the details are completely different. ${ }^{38}$ The Lines approach requires an accurate description of the crystal field; however, if accurate crystal field parameters are available, the Hamiltonian may be diagonalized directly, as described above. The quantitative diamagnetic substitution approach can be used to quantify the ground state exchange coupling when the crystal field parameters are not available. The following sections provide the basis for this approach.

\section{Susceptibility of a magnetically isolated $f$-ion}

The method described here extracts crystal field information from the susceptibility of a magnetically isolated $f$-ion; therefore, the starting point is to describe the susceptibility of an isolated $f$-ion. The $f$-ion is assumed to possess axial symmetry. While it is possible to perform this analysis in lower symmetry, the majority of the reported complexes where this method can be applied possess axial or nearly axial symmetry. For an isolated $f$-metal ion, a crystal field of axial (or lower) symmetry will split the ${ }^{2 \mathrm{~S}+1} \mathrm{~L}_{\eta}$ free-ion terms into a number of states characterized by $\mathrm{m}_{\mathrm{J}}$ (in reality, the states will generally be some linear combination of $\mathrm{m}_{\mathrm{J}}$ states). ${ }^{28}$ If the ion possesses an odd number of electrons, an axial crystal field will split each ${ }^{2 \mathrm{~S}+1} \mathrm{~L}_{\eta}$ term into $\eta+1 / 2$ 
Kramers' doublets, and if the ion possesses an even number of electrons, most of these states are doublets, but at least one state is a singlet. ${ }^{28}$ These doublets and singlets can be described using a fictitious effective spin, $\tilde{\mathrm{S}}$, equal to $1 \frac{1}{2}$ for the doublets and zero for the singlets. The splitting of each effective-spin doublet in a magnetic field will be anisotropic due to the axial crystal field, and can be described using $g_{z}$ and $g_{x}$ to represent the splitting when the magnetic field is parallel and perpendicular to the principal crystal field axis, respectively. ${ }^{28}$ The susceptibility of the $f$-ion may be represented by eq 1 where $\tilde{S}_{n}, \mathbf{g}_{n}, E_{n}$, and $Z_{n}$ are the effective spin, g-values of the effective spins, the energy, and the second-order Zeeman term of the $\mathrm{n}^{\text {th }}$ state, respectively, and the other symbols have their usual meanings. ${ }^{39}$ Since the states are typically quite anisotropic, the susceptibility along each axis is treated separately, and the powder susceptibility is the average of the susceptibility along each axis.

$$
\chi_{\text {iso, } \alpha}=\frac{N \beta^{2}}{3 k T} \frac{\sum_{n}\left(2 \tilde{S}_{n}+1\right)\left[\tilde{S}_{n}\left(\tilde{S}_{n}+1\right) g_{n, \alpha}^{2}-2 Z_{n} k T\right] e^{-E_{n} / k T}}{\sum_{n}\left(2 \tilde{S}_{n}+1\right) e^{-E_{n} / k T}} \alpha=x \text { or } z
$$

\section{Exchange coupling of thermally isolated doublets and singlets}

The goal of the quantitative diamagnetic substitution method is to quantify the exchange coupling of the ground state doublets of the $f$-ions. For this reason, the exchange coupling in such an doublet, isolated from the excited states, will be examined. The phenomenological Heisenberg-Dirac-Van Vleck Hamiltonian for exchange coupling of the spins of the $f$-electrons of a pair of identical, exchange-coupled $f$-ions related by an inversion center is given by eq 2 , where $\mathbf{J}$ is diagonal, which may be rewritten as eq 3 where $\mathbf{J}$ is the isotropic term and $\boldsymbol{\Lambda}$ is the 
traceless anisotropy tensor with $\Lambda_{\mathrm{xx}}=\Lambda_{\mathrm{yy}}=-\Lambda_{\mathrm{zz}} / 2$ and the off-diagonal elements are zero under axial symmetry. ${ }^{28,38,40}$ The spins of the $f$-electrons will be referred to as "true spins" to distinguish them from the effective spins. Antisymmetric exchange is not included since the $f$-ions are related by an inversion center. ${ }^{41}$

$$
\begin{aligned}
& \mathbb{Z}_{\mathrm{ex}}=-2 \mathbf{S}_{\mathrm{a}} \cdot \mathbf{J} \cdot \mathbf{S}_{\mathrm{b}}(2) \\
& \mathcal{H}_{\mathrm{ex}}=-2 J \mathbf{S}_{\mathrm{a}} \cdot \mathbf{S}_{\mathrm{b}}-\mathbf{S}_{\mathrm{a}} \cdot \Lambda \cdot \mathbf{S}_{\mathrm{b}}
\end{aligned}
$$

Assuming Russel-Saunders coupling, the true spin may be projected onto the angular momentum, q, using $S=\left(g_{J}-1\right)$ g, where $g_{J}$ is the Landé g-value. ${ }^{28,42}$ The addition of the Zeeman term to the exchange term gives the spin Hamiltonian for the exchange-coupled system, eq 4.

$$
\mathbb{Z}^{4}=\mathrm{g}_{\mathrm{J}} \beta\left(\mathbf{H} \cdot \mathbf{J}_{\mathrm{a}}+\mathbf{H} \cdot \mathbf{J}_{\mathrm{b}}\right)-\left(\mathrm{g}_{\mathrm{J}}-1\right)^{2}\left(2 \mathrm{~J} \mathbf{J}_{\mathrm{a}} \cdot \mathbf{J}_{\mathrm{b}}+\mathbf{J}_{\mathrm{a}} \cdot \boldsymbol{\Lambda} \cdot \mathbf{J}_{\mathrm{b}}\right)
$$

As in the magnetically isolated case, the axial crystal field splits the ${ }^{2 \mathrm{~S}+1} \mathrm{~L}_{\eta}$ term into a number of states, each of which can be described by an effective spin, $\tilde{\mathrm{S}}$, which, is equivalent to $\mathrm{g}_{\mathrm{J}} \mathrm{q}_{\alpha} / \mathrm{g}_{\alpha}$, where $\alpha$ is $\mathrm{z}, \mathrm{y}$, or $\mathrm{z}^{28}$ The effective-spin Hamiltonian for two identical interacting $f$-ions is then given by eq 5 , which includes the dipole-dipole coupling term, $\mathbf{D}$, where $\mathrm{D}_{\mathrm{xx}}=\mathrm{D}_{\mathrm{yy}}=-\mathrm{D}_{\mathrm{zz}} / 2, \mathrm{D}_{\mathrm{zz}}=$ $2 \mathrm{D}_{\mathrm{dd}} / 3$, and $\mathrm{D}_{\mathrm{dd}}$ is the magnitude of the dipole-dipole interaction and the $\mathrm{z}$-axis is the intermetallic axis as well as the axis of symmetry. ${ }^{28}$ If the symmetry of the complex is lower than axial, $g_{z}$ may not be coincident with the intermetallic axis, and eqs 7 and 8 would need to be changed accordingly. Equation 5 may be more conveniently expressed as the Hamiltonian for 
anisotropic coupling, eq 6 , where $\mathrm{J}_{\mathrm{x}}, \mathrm{J}_{\mathrm{z}}$, and $\mathrm{D}_{\mathrm{dd}}$ are given by eqs $7-9$ and $\beta^{2} / \mathrm{r}^{3}=4.4 \times 10^{-4} \mathrm{~cm}^{-1}$ $\mathrm{nm}^{-3}$.

$$
\tilde{\mathcal{H}}=\beta\left(\mathbf{H} \cdot \mathbf{g} \cdot \tilde{\mathbf{S}}_{\mathrm{a}}+\mathbf{H} \cdot \mathbf{g} \cdot \tilde{\mathbf{S}}_{\mathrm{b}}\right)-\frac{\left(\mathrm{g}_{\mathrm{J}}-1\right)^{2}}{\mathrm{~g}_{\mathbf{J}}^{2}}\left(2 \mathrm{~J} \tilde{\mathbf{S}}_{\mathrm{a}} \cdot \mathbf{g}^{2} \cdot \tilde{\mathbf{S}}_{\mathrm{b}}+\tilde{\mathbf{S}}_{\mathrm{a}} \cdot \mathbf{g}^{2} \cdot \mathbf{\Lambda} \cdot \tilde{\mathbf{S}}_{\mathrm{b}}\right)+\tilde{\mathbf{S}}_{\mathrm{a}} \cdot \mathbf{D} \cdot \tilde{\mathbf{S}}_{\mathrm{b}}
$$

$$
\tilde{\mathcal{H}}=\beta\left(\mathbf{H} \cdot \mathbf{g} \cdot \tilde{\mathbf{S}}_{\mathrm{a}}+\mathbf{H} \cdot \mathbf{g} \cdot \tilde{\mathbf{S}}_{\mathrm{b}}\right)-2 \mathrm{~J}_{\mathrm{z}}\left(\tilde{\mathrm{S}}_{\mathrm{za}} \tilde{\mathrm{S}}_{\mathrm{zb}}\right)-2 \mathrm{~J}_{\mathrm{x}}\left(\tilde{\mathrm{S}}_{\mathrm{xa}} \tilde{\mathrm{S}}_{\mathrm{xb}}+\tilde{\mathrm{S}}_{\mathrm{ya}} \tilde{\mathrm{S}}_{\mathrm{yb}}\right)
$$

$$
\mathrm{J}_{\mathrm{Z}}=\frac{\mathrm{D}_{\mathrm{dd}}}{3}+\frac{\mathrm{g}_{\mathrm{Z}}^{2}\left(\mathrm{~g}_{\mathrm{J}}-1\right)^{2}}{\mathrm{~g}_{\mathrm{J}}^{2}}\left(\mathrm{~J}+\frac{\Lambda_{\mathrm{zZ}}}{2}\right)=\frac{\mathrm{D}_{\mathrm{dd}}}{3}+\frac{\mathrm{g}_{\mathrm{Z}}^{2}\left(\mathrm{~g}_{\mathrm{J}}-1\right)^{2}}{\mathrm{~g}_{\mathrm{J}}^{2}}\left(\mathrm{~J}-\Lambda_{\mathrm{Xx}}\right)
$$

$$
\mathrm{J}_{\mathrm{x}}=-\frac{\mathrm{D}_{\mathrm{dd}}}{6}+\frac{\mathrm{g}_{\mathrm{x}}^{2}\left(\mathrm{~g}_{\mathrm{J}}-1\right)^{2}}{\mathrm{~g}_{\mathrm{J}}^{2}}\left(\mathrm{~J}+\frac{\Lambda_{\mathrm{xx}}}{2}\right)(8)
$$

$$
D_{d d}=\left(2 g_{z}^{2}+g_{x}^{2}\right) \frac{\beta^{2}}{2 r^{3}}
$$

For this effective spin Hamiltonian, the magnetic susceptibility is given by eqs 10 and 12 . Different forms of these equations have been reported by Nakatsuka, et al. for antiferromagnetic coupling. ${ }^{43}$ Since $\mathrm{F}_{\alpha}(\mathrm{J}, \mathrm{g}, \mathrm{T})$ becomes 2 as $\mathrm{J}$ goes to zero, the susceptibility of uncoupled $f$-ions is just the sum of the susceptibilities of the isolated $f$-ions. Therefore, for the singlet states, which are not exchange-coupled, the susceptibility of the coupled pair is equal to the sum of the susceptibilities of the isolated states. 


$$
\begin{aligned}
& \chi_{\alpha}=\frac{\tilde{S}_{n}\left(\tilde{S}_{n}+1\right) N \beta^{2} g_{\alpha}^{2}}{3 k T} F_{\alpha}(J, g, T) \quad \alpha=x \text { or } z \quad(10) \\
& F_{Z}(J, g, T)=\frac{4}{1+e^{-J_{Z} / k T} \cosh \left(J_{X} / k T\right)} \quad(11) \\
& F_{X}(J, g, T)=\frac{4 k T \sinh \left[\left(J_{Z}-J_{x}\right) / 2 k T\right]}{\left(J_{Z}-J_{X}\right)\left\{\cosh \left[\left(J_{Z}-J_{x}\right) / 2 k T\right]+e^{-J_{x} / k T} \cosh \left[\left(J_{Z}+J_{x}\right) / 2 k T\right]\right\}}
\end{aligned}
$$

\section{Susceptibility of a pair of coupled $f$-ions}

Figure 1 is a qualitative diagram illustrating the relationship of the low-lying states of a pair of coupled $f$-ions to the states of the isolated $f$-ions along a given axis. Since $\mathbf{g}_{\mathrm{n}}$ is typically anisotropic, the splitting of the states will be anisotropic even when the exchange of the true spins is isotropic. The susceptibility of the coupled pair can be described by combining eq 1 with eq 10 to give eq 13 .

$$
\chi_{\alpha, \text { pair }}=\frac{N \beta^{2}}{3 k T} \frac{\sum_{n} F_{\alpha}\left(J_{n}, g_{n}, T\right)\left(2 \tilde{S}_{n}+1\right)\left[\tilde{S}_{n}\left(\tilde{S}_{n}+1\right) g_{n, \alpha}^{2}-2 Z_{n} k T\right] e^{-E_{n} / k T}}{\sum_{n}\left(2 \tilde{S}_{n}+1\right) e^{-E_{n} / k T}}(\alpha=x \text { or } z)
$$




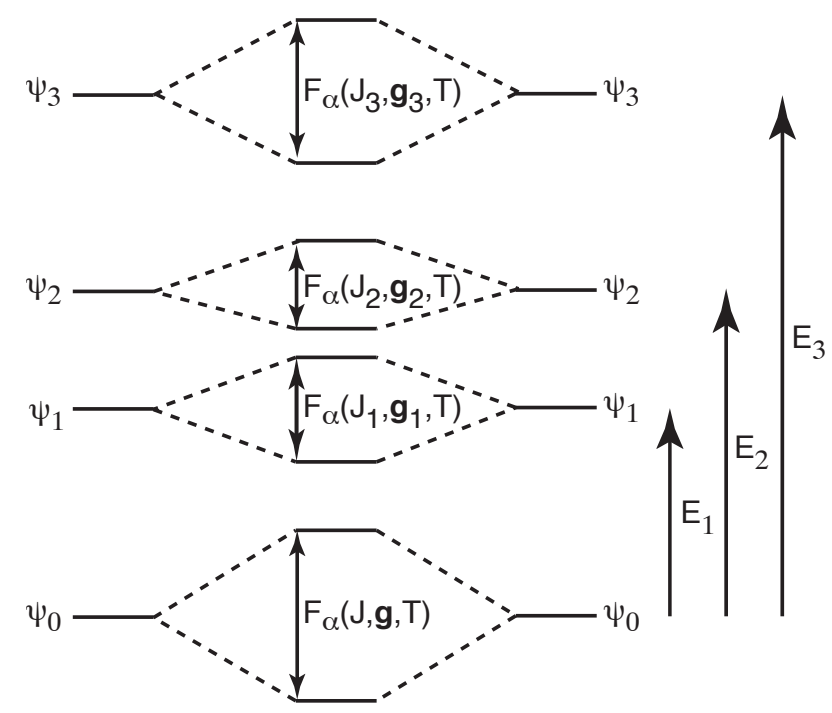

Figure 1. Qualitative diagram illustrating the splitting of the low-lying states of a pair of coupled $f$-ions along a given axis, $\alpha$, where $\alpha$ is $\mathrm{x}, \mathrm{y}$, or $\mathrm{z}$. The splitting due to coupling is exaggerated for clarity; $\mathrm{J}$ is generally much smaller than $\mathrm{E}_{\mathrm{n}}$.

\section{Approximations}

While eq 13 describes the susceptibility of a pair of identical $f$-ions undergoing exchange coupling, it is not particularly useful if the values of $\tilde{S}_{n}, g_{n}, E_{n}$, and $Z_{w, n}$ are unknown. In other words, it is not useful if the crystal field parameters are unknown. Moreover, if the crystal field parameters are known, the more appropriate approach would be to diagonalize the full Hamiltonian, as described in the introduction. Nevertheless, eq 13 may be used to quantify coupling of the ground state doublet using the diamagnetic substitution method with certain approximations. In order to determine when this approach is accurate, it is necessary to examine the approximations necessary to apply the diamagnetic substitution method and determine when they are valid. 
The first approximation is that the exchange coupling of the excited states is identical to that of the ground state. In other words, the $\mathrm{F}_{\alpha}\left(\mathrm{J}_{\mathrm{n}}, \mathrm{g}_{\mathbf{n}}, \mathrm{T}\right)$ terms in eq 13 are replaced by $\mathrm{F}_{\alpha}(\mathrm{J}, \mathbf{g}, \mathrm{T})$ of the ground state doublet to give eq 14. This approximation is valid when the energy of the first excited state is much greater than the exchange coupling $\left(E_{n} \gg>J_{n}\right)$, which is usually true if the crystal field is much stronger than the exchange coupling. In this case, at the temperatures at which the $\mathrm{n}^{\text {th }}$ state is occupied, $\mathrm{F}_{\alpha}\left(\mathrm{J}_{\mathrm{n}}, \mathrm{g}_{\mathrm{n}}, \mathrm{T}\right)$ has only a small effect on the susceptibility of the pair, so replacing it with ground state splitting, $\mathrm{F}_{\alpha}(\mathrm{J}, \mathbf{g}, \mathrm{T})$, is a good approximation.

$$
\chi_{\alpha, \text { pair }} \cong F_{\alpha}(\mathrm{J}, \mathbf{g}, \mathrm{T})\left\{\frac{\mathrm{N} \beta^{2}}{3 \mathrm{kT}} \frac{\sum_{\mathrm{n}}\left(2 \tilde{\mathrm{S}}_{\mathrm{n}}+1\right)\left[\tilde{\mathrm{S}}_{\mathrm{n}}\left(\tilde{\mathrm{S}}_{\mathrm{n}}+1\right) \mathrm{g}_{\mathrm{n}, \alpha}^{2}-2 \mathrm{Z}_{\mathrm{n}} \mathrm{kT}\right] \mathrm{e}^{-\mathrm{E}_{\mathrm{n}} / \mathrm{kT}}}{\sum_{\mathrm{n}}\left(2 \tilde{\mathrm{S}}_{\mathrm{n}}+1\right) \mathrm{e}^{-\mathrm{E}_{\mathrm{n}} / \mathrm{kT}}}\right\}(\alpha=\mathrm{x} \text { or } \mathrm{z})
$$

The second approximation is that crystal field of the $f$-ion in the coupled complex is the same as that of the magnetically isolated analog. Therefore, the term in braces in eq 14 can be replaced by the susceptibility of the magnetically isolated analog yielding eq 15 . The validity of this approximation is determined by the similarity of the ligand environment of the magnetically isolated analog to that of the coupled complex. There are many examples for which this approximation, as well as the preceding one, is valid since these are the requirements for using the diamagnetic substitution method..$^{1,18-23,26}$

$$
\chi_{\alpha, \text { pair }} \cong \mathrm{F}_{\alpha}(\mathrm{J}, \mathbf{g}, \mathrm{T}) \chi_{\alpha, \text { iso }}
$$


A final approximation is needed to approximate $\chi_{\alpha, \text { iso }}$ from the powder susceptibility of the magnetically isolated analog $\left(\chi_{\alpha, \text { iso }}\right.$ can be determined by single-crystal susceptibility with no assumptions). It is necessary to define a single-ion anisotropy parameter, $\gamma$, where $\gamma=g_{x} / g_{z}$ and $g_{z}$ and $g_{x}$ are the g-values for the ground state doublet of the magnetically isolated analog. For $f$ ions with an even number of electrons, $g_{x}$ and $\gamma$ are zero except in rare cases ${ }^{28}$ For $f$-ions with an odd number of electrons, $\gamma$ can be determined from by $g_{z}$ and $g_{x}$, which can be measured by EPR spectroscopy at low temperature. If a low temperature EPR spectrum cannot be observed, $\mathrm{g}_{\mathrm{x}}$ must be zero (or very small), so $\gamma$ is zero (or very close to it). ${ }^{28}$ Alternatively, $\gamma$ can be determined from the wavefunction of the ground state..$^{44,45}$ The third and final approximation is that the anisotropy of all of the energy levels is equal to that of the ground state doublet, $\gamma$. For ions with an even number of electrons, this approximation is generally true since $\gamma$ is usually zero. For ions with an odd number of electrons, this approximation is certainly false, but is still valid when the energy of the first excited state is much greater than the exchange coupling for the reason discussed in the first approximation.

Since the anisotropy of the excited states can be approximated using $\gamma, \chi_{\alpha \text {,iso }}$ can be approximated from $\gamma$ and the powder susceptibility of a magnetically isolated analog, $\chi_{\text {iso }}$, using eqs 16 and 17 at each temperature. The susceptibility of the coupled system can then be simulated using eq 15 and allowing $\mathrm{J}$ to vary since $\mathrm{J}_{\mathrm{z}}$ and $\mathrm{J}_{\mathrm{x}}$ are given by eqs 10 and 11 . As shown below, allowing the anisotropic coupling of the true spins, $\Lambda_{\mathrm{xx}}$, to vary is problematic, so it should be determined independently. 


$$
\begin{aligned}
& \chi_{\text {z,iso }} \approx \frac{3 \chi_{\text {iso }}}{1+2 \gamma^{2}} \\
& \chi_{\text {x,iso }} \approx \gamma^{2} \chi_{\text {z,iso }}
\end{aligned}
$$

The main result of the methodology described above is that the exchange coupling, $\mathbf{J}$, of the $f$ electrons (the true spins) and the coupling parameters for the ground state doublet, $\mathrm{J}_{\mathrm{x}}$ and $\mathrm{J}_{\mathrm{z}}$, may be determined from the magnetic susceptibility of a pair of identical exchange-coupled $f$-ions without knowing the crystal field parameters. For the ground state, the only assumption is that the crystal field of the $f$-ions in the coupled system is the same as that in the magnetically isolated analog; the other assumptions apply only to the excited states. As shown above, this approach is valid if the energy of the first excited state is much greater than the exchange coupling and if the crystal field of the coupled complex is sufficiently similar to that of the magnetically isolated analog.

\section{Application to $f$-ion systems}

The formulas given above describe the results for an ideal experiment. In practice, two experimental problems need to be addressed: paramagnetic impurities and weighing errors. The influence of paramagnetic impurities are not always readily apparent in the susceptibility plots; however, their inclusion does dramatically affect the fit in some cases, and previous work in related systems clearly shows that paramagnetic impurities may be present. ${ }^{6,81}$ The other issue is weighing error, which is particularly problematic for air sensitive samples due to charging and buoyancy effects (in spin-only models, of allowing g to vary as a fitting parameter mitigates any weighing errors). For these reasons, the data described below was modeled using eq 18, where 
$\chi_{\mathrm{x}, \mathrm{pair}}$ and $\chi_{\mathrm{z} \text {,pair }}$ are given by eq $15, \mathrm{C}$ is the Curie constant for the impurities and $\mathrm{w}$ is the factor to correct for weighing errors and should be roughly equal to 1 . All fits were initially conducted with $\mathrm{w}$ and $\mathrm{C}$ equal to 1 and 0 , respectively, then $\mathrm{w}$ and $\mathrm{C}$ were allowed to vary. If inclusion of $\mathrm{w}$ and/or $\mathrm{C}$ did not decrease chi-squared by more than $10 \%$, they were fixed at 1 and 0 , respectively. Empirically, allowing $\mathrm{w}$ and $\mathrm{C}$ to vary has very little effect on $\mathrm{J}$.

$\chi_{\text {calc }}=\mathrm{w}\left(\frac{\chi_{z, \text { pair }}+2 \chi_{x, \text { pair }}}{3}\right)+\frac{\mathrm{C}}{\mathrm{T}}$

Lanthanide phthalocyanine triple-deckers, $\mathrm{M}_{2} \mathrm{Pc}_{3}$, where $\mathrm{M}=\mathrm{Tb}, \mathrm{Dy}, \mathrm{Ho}, \mathrm{Er}$ and $\mathrm{Pc}=$ phthalocyanine

This first example compares the results of the quantitative diamagnetic substitution method to the results of a detailed calculation using crystal field parameters. To the best of our knowledge, the only system of $f$-ion pair for which detailed calculations and the susceptibilities of the coupled systems as well as a magnetically isolated analogs are available is a series of late lanthanide phthalocyanine complexes studied by Ishikawa, et al. for Tb through $\mathrm{Yb}$. $^{10-13,46}$ The coupling between the $f$-ions in these complexes was examined in detail and found to originate almost exclusively from dipole-dipole coupling. The authors provide an ideal set of magnetically isolated analogs by replacing one of the lanthanide ions with a nonmagnetic yttrium ion, and they report the ground state assignments for these molecules from which $\gamma$ and $\mathrm{g}_{\mathrm{z}}$ were determined. The magnetic susceptibilities of the triple-deckers are shown in Figure 1 along with the leastsquares fits using eq 18. The results of fitting the data are reported in Table 1 . In all cases, the coupling between the $f$-ions in these late lanthanide complexes is largely due to dipole-dipole 
interactions, and the contribution from exchange coupling is minor at best, which is in good agreement with the detailed calculations reported by Ishikawa, et al. ${ }^{11}$
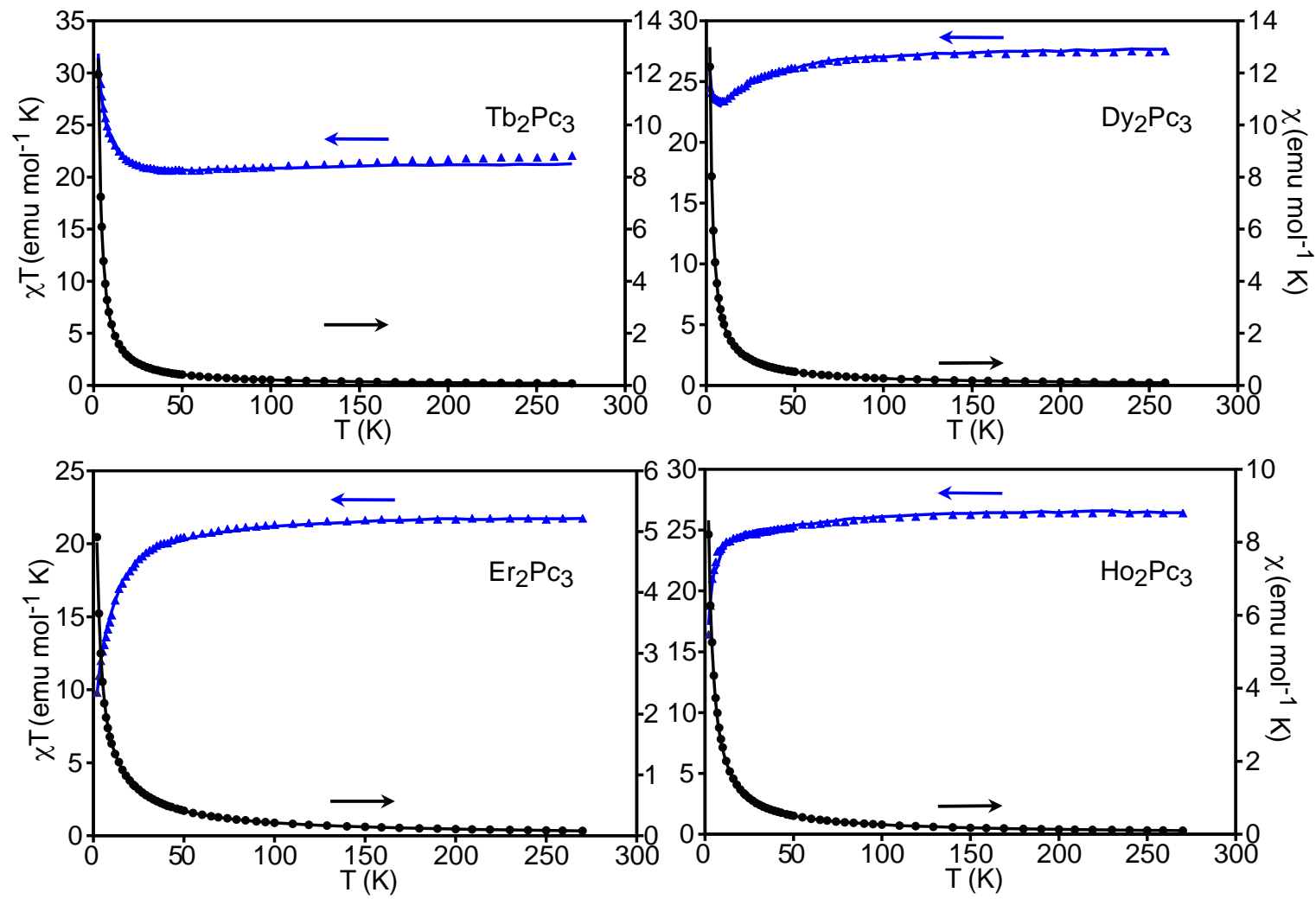

Figure 2. Magnetic susceptibility of lanthanide phthalocyanine triple-decker complexes (symbols) and fits (lines) performed using eqs 18 and the anisotropy and susceptibility of the magnetically isolated analogs, $\mathrm{MYPc}_{3}$, prepared by replacing one of the ions with yttrium (M=Tb-Er). Susceptibility data are taken from ref. 11 . 
Table 1: Data for coupling between $f$-element pairs (only $\mathrm{J}, \mathrm{w}$, and $\mathrm{C}$ are variables)

\begin{tabular}{|c|c|c|c|c|c|c|c|c|c|}
\hline Compound & $\gamma^{\mathrm{a}}$ & $\mathrm{g}_{\mathrm{z}}{ }^{\mathrm{a}}$ & $r(\AA)$ & $\begin{array}{c}\mathrm{D}_{\mathrm{dd}} \\
\left(\mathrm{cm}^{-1}\right)\end{array}$ & $\begin{array}{c}\Lambda_{\mathrm{xx}} \\
\left(\mathrm{cm}^{-1}\right)\end{array}$ & $\begin{array}{c}\mathrm{J} \\
\left(\mathrm{cm}^{-1}\right)\end{array}$ & $\begin{array}{c}\mathrm{J}_{\mathrm{z}}, \mathrm{J}_{\mathrm{x}} \\
\left(\mathrm{cm}^{-1}\right)\end{array}$ & w & $\mathrm{C}$ \\
\hline $\mathrm{Pc}_{3} \mathrm{~Tb}_{2}{ }^{11}$ & 0 & 17.65 & 3.57 & 3.0 & 0 & 0.07 & $3.2,-0.49$ & 0.94 & $0^{\mathrm{b}}$ \\
\hline $\mathrm{Pc}_{3} \mathrm{Dy}_{2}{ }^{11}$ & 0 & 16.0 & 3.57 & 2.0 & 0 & 0.08 & $1.7,-0.33$ & $1^{\mathrm{b}}$ & $0^{\mathrm{b}}$ \\
\hline $\mathrm{Pc}_{3} \mathrm{Ho}_{2}{ }^{11}$ & 0 & 13.1 & 3.57 & 1.6 & 0 & -0.04 & $0.25,-0.27$ & $1^{\mathrm{b}}$ & $0^{\mathrm{b}}$ \\
\hline $\mathrm{Pc}_{3} \mathrm{Er}_{2}{ }^{11}$ & 8.08 & 1.57 & 3.57 & 0.8 & 0 & -0.05 & $0.26,-0.36$ & $1^{\mathrm{b}}$ & $0^{\mathrm{b}}$ \\
\hline $\mathrm{COT}_{3} \mathrm{Ce}_{2}^{7}$ & 2.023 & 1.123 & 4.0 & 0.026 & $-0.035^{c}$ & -35 & $-1.2,-5.1$ & 0.95 & 0.029 \\
\hline $\begin{array}{l}{\left[\mathrm{MeCp} \mathrm{p}_{3} \mathrm{U}\right]_{2}} \\
\left(\mu-\mathrm{N}-\mathrm{C}_{6} \mathrm{H}_{4}-\mathrm{N}\right)^{31}\end{array}$ & 0 & 2.57 & $9.8^{\mathrm{d}}$ & 0.003 & 0 & -90 & $-16,-5 \times 10^{-4}$ & 0.92 & 0.029 \\
\hline $\begin{array}{l}{\left[\mathrm{Cp}_{2}^{*} \mathrm{Yb}\right]_{2}} \\
(\mu \text {-bipyrimidine })^{35}\end{array}$ & 0.205 & 7.050 & 6.23 & 0.09 & 0 & -13 & $-10,-0.45$ & 1.02 & $0^{\mathrm{b}}$ \\
\hline $\begin{array}{l}{\left[\mathrm{Cp} *_{2} \mathrm{Yb}\right]_{2}} \\
(\mu \text {-azobenzene })^{35}\end{array}$ & 0.205 & 7.050 & 9.17 & 0.03 & 0 & -17 & $-14,-0.56$ & 1.06 & 0.055 \\
\hline
\end{tabular}

a) From EPR for $\left[\mathrm{COT}_{2} \mathrm{Ce}\right]^{-}$and the $\left[\mathrm{Cp}^{*}{ }_{2} \mathrm{Yb}\right](\mu-\mathrm{L})$ complexes and calculated from the ground state for the others.

b) Not varied.

c) From EPR for $\mathrm{COT}_{3} \mathrm{Ce}_{2}$.

d) Estimated from the crystal structure of 1,4-diaminobenzene and the U-N distance in $(\mathrm{MeCp})_{3} \mathrm{UNC}_{5} \mathrm{H}_{6}{ }^{47,48}$ 


\section{Cerium cyclooctatetraene triple-decker, $\mathrm{Ce}_{2} \mathrm{COT}_{3}\left(\mathrm{COT}=\mathrm{C}_{8} \mathrm{H}_{8}\right)$}

The magnetism of this complex has previously been reported by Walter, et al.; however, the coupling was not correctly modeled. ${ }^{7}$ In the previous report, a version of the method described here was applied directly to the effective spins rather than to the true spins. Consequently, $\chi_{\text {iso }}$ was inserted into an equation describing the coupling between the effective spins as isotropic rather than highly anisotropic.

$\mathrm{Ce}_{2} \mathrm{COT}_{3}$ is a particularly useful example since both it and its magnetically isolated analog, anionic $\left[\mathrm{COT}_{2} \mathrm{Ce}\right]^{-}$, are EPR active. The anisotropy $(\gamma), 2.023$, and value of $\mathrm{g}_{\mathrm{z}}, 1.123$, were determined from the EPR spectrum of $\left[\mathrm{COT}_{2} \mathrm{Ce}\right]^{-}$. The value of $\Lambda_{\mathrm{xx}}$ was determined from the EPR spectrum of $\mathrm{Ce}_{2} \mathrm{COT}_{3}$. The observed magnitudes of the zero-field splitting of the effective spin triplet state of $\mathrm{Ce}_{2} \mathrm{COT}_{3}$ are $\mathrm{D}_{\mathrm{x}}=0.014 \mathrm{~cm}^{-1}$ and $\mathrm{D}_{\mathrm{z}}=0.020 \mathrm{~cm}^{-1}$, which must have opposite signs, and $\mathrm{D}_{\mathrm{z}}$ is assumed to be positive. These may be corrected for the effect of dipole-dipole coupling to give, $\mathrm{D}_{\mathrm{x}}{ }^{\prime}=-5.3 \times 10^{-3} \mathrm{~cm}^{-1}$ and $\mathrm{D}_{\mathrm{z}}{ }^{\prime}=2.7 \times 10^{-3} \mathrm{~cm}^{-1}$ from which $\Lambda_{\mathrm{xx}}$ and $\Lambda_{\mathrm{zz}}$, may be determined using $\mathrm{g}_{\mathrm{J}}, \mathrm{g}_{\mathrm{x}}$, and $\mathrm{g}_{\mathrm{z}}$ to give $-3.7 \times 10^{-2} \mathrm{~cm}^{-1}$ and $7.7 \times 10^{-2} \mathrm{~cm}^{-1}$, respectively, which are in good agreement with the requirement that $\Lambda_{\mathrm{xx}}=-\Lambda_{\mathrm{zz}} / 2$. Eq 15 can be used to fit the susceptibility of $\mathrm{Ce}_{2} \mathrm{COT}_{3}$ with $\mathrm{J}$ as the only variable (Figure $\mathrm{S} 1$ ); however, a better fit, shown in Figure 3, may be obtained using eq 18 with the values given in Table 1. 


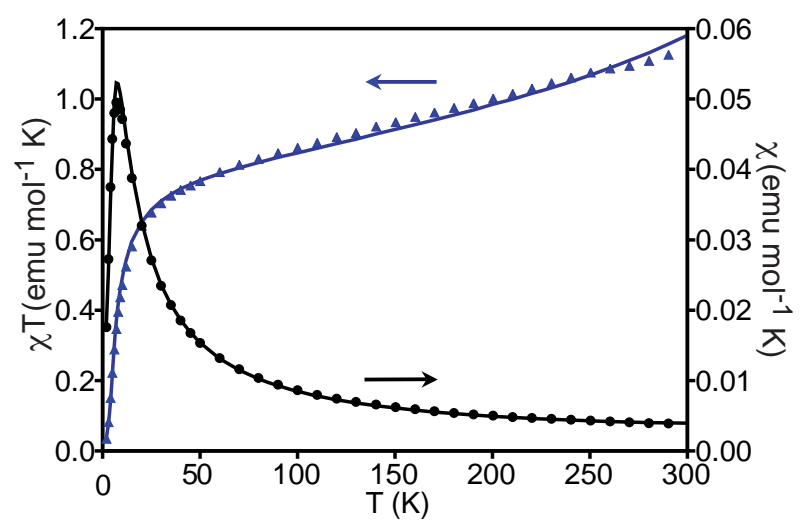

Figure 3. Magnetic susceptibility of $\mathrm{Ce}_{2} \mathrm{COT}_{3}$ (symbols) and fit (lines) performed using eq 18 and the anisotropy and susceptibility of the magnetically isolated analog $\left[\mathrm{COT}_{2} \mathrm{Ce}\right]^{-}$. Susceptibility data are taken from ref. 7.

The isotropic exchange coupling is much stronger than either the dipole-dipole coupling or the anisotropic coupling. The weighted average of the phenomenological parameters, $\left(\mathrm{J}_{\mathrm{z}}+2 \mathrm{~J}_{\mathrm{x}}\right) / 3$, is $3.8 \mathrm{~cm}^{-1}$, which is similar to that previously reported isotropic value, $-4.0 \mathrm{~cm}^{-1}$. However, the coupling of the true spins is much stronger than that of the effective spins. As shown in Figure 3 , the fit is generally good with some deviation at high temperature. The deviation is believed to be a consequence of the shorter COT-Ce distance in $\left[\mathrm{COT}_{2} \mathrm{Ce}\right]^{-}$than in $\mathrm{COT}_{3} \mathrm{Ce}_{2}(2.68$ vs. $2.73 \AA$ ) resulting in a stronger crystal field in $\left[\mathrm{COT}_{2} \mathrm{Ce}\right]^{-}$. The assumption that the crystal field in the magnetically isolated analog is identical to the coupled complex is therefore not completely true; however, it is still fairly good.

As noted above, allowing the coupling anisotropy, $\Lambda_{\mathrm{xx}}$, to vary as a fitting parameter is problematic. In this case, $\Lambda_{\mathrm{xx}}$ can be determined from the EPR spectrum of $\mathrm{COT}_{3} \mathrm{Ce}_{2}$, and $\mathrm{J}$ can be determined by fitting the susceptibility. If both $\mathrm{J}$ and $\Lambda_{\mathrm{xx}}$ are allowed to vary when fitting the 
susceptibility, a slightly better fit is obtained with $\mathrm{J}$ and $\Lambda_{\mathrm{xx}}$ equal to $-14 \mathrm{~cm}^{-1}$ and $-60 \mathrm{~cm}^{-1}$, respectively; however, this value of $\Lambda_{\mathrm{xx}}$ is clearly incorrect. In the complexes examined here, the major contributor to the anisotropy of the coupling is the single-ion anisotropy due to coupling of the spins of the $f$-electrons with their unquenched orbital angular momenta. It should be noted the anisotropy of the coupling can be significant for systems in which $f$-ions interact with transition metals. $^{29}$

\section{A U(V) pair: $\left[(M e C p)_{3}\right]_{2}\left(\mu-1,4-\mathbf{N}_{2} C_{6} H_{4}\right)$}

This molecule, reported by Rosen, et al., is one of the archetypical examples of exchange coupling between $f$-metal ions. ${ }^{31}$ The anisotropy and value of $\mathrm{g}_{\mathrm{z}}$ were determined from the failure to observe an EPR spectrum for this complex or for the monomeric $(\mathrm{MeCp})_{3} \mathrm{UNC}_{6} \mathrm{H}_{5}$. The ground state must be ${ }^{2} \mathrm{~F}_{5 / 2}$ with $\mathrm{m}_{\mathrm{J}}= \pm 3 / 2$, for which $\gamma$ is zero and $\mathrm{g}_{\mathrm{z}}$ is 2.57 . ${ }^{31}\left[(\mathrm{MeCp})_{3} \mathrm{U}\right]_{2}(\mu-1,3$ $\mathrm{N}_{2} \mathrm{C}_{6} \mathrm{H}_{4}$ ) was used as the magnetically isolated analog (the uranium centers are not appreciably coupled when bridged by $1,3-\mathrm{N}_{2} \mathrm{C}_{6} \mathrm{H}_{4}$ ). The susceptibility of the coupled sample, shown in Figure 4, was fit using eq 18, and the fit parameters are given in Table 1. As in the original report, an impurity contribution had to be included to obtain an acceptable fit. In this reexamination, the best fit to the data was provided by a simple paramagnet rather than a uranium impurity. The value of $\mathrm{J}_{\mathrm{z}}$ is similar to the previously reported phenomenological coupling constant. 


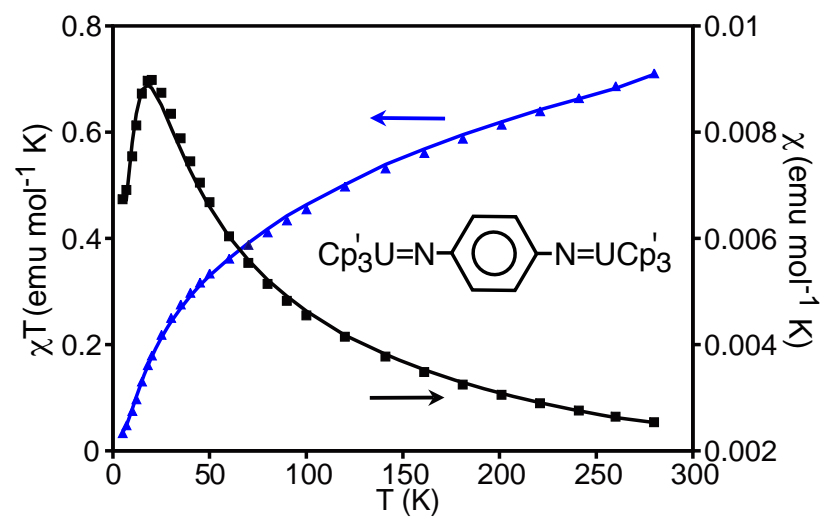

Figure 4. Magnetic susceptibility (symbols) of $\left[(\mathrm{MeCp})_{3} \mathrm{U}\right]_{2}\left(\mu-1,4-\mathrm{C}_{6} \mathrm{H}_{4} \mathrm{~N}_{2}\right)$ and fits (lines) performed using eqs 18 and the anisotropy and susceptibility of the magnetically isolated analog $\left[(\mathrm{MeCp})_{3} \mathrm{U}\right]_{2}\left(\mu-1,3-\mathrm{C}_{6} \mathrm{H}_{4} \mathrm{~N}_{2}\right)$. Susceptibility data are taken from ref. 31 .

Decamethylytterbocene complexes: $\left[\mathrm{Cp}_{2}{ }_{2} \mathrm{Yb}\right]_{2}(\mu-\mathrm{L})$, where $\mathrm{L}=2$,2'-bipyrimidine and azobenzene, and $\mathrm{Cp}^{*}=$ pentamethylcyclopentadienyl

A series of complexes consisting of two $\mathrm{Cp}^{*}{ }_{2} \mathrm{Yb}$ units linked by a heterocyclic nitrogen bases were described by Berg, et al. ${ }^{35}$ These complexes show evidence for exchange coupling between the $\mathrm{Yb}(\mathrm{III})$ centers, which is surprising in light of the fact that the $4 f$ orbitals of $\mathrm{Yb}(\mathrm{III})$ are highly contracted and not anticipated to sufficiently participate in bonding to allow superexchange via covalency. The complex with the weakest coupling is $\left[\mathrm{Cp}_{2}{ }_{2} \mathrm{Yb}\right]_{2}(\mu$-bipm) (bipm is 2,2'dipyrimidine), which is of particular interest due to the similarity of the $\mathrm{Yb}$ coordination environment to that of $\mathrm{Cp}_{2}{ }_{2} \mathrm{Yb}$ (bipy) (bipy is 2,2'-bipyridine) for which extensive data is available. $^{6,8}$ Consequently, it was assumed that the magnetically isolated analog of $\left[\mathrm{Cp}_{2}{ }_{2} \mathrm{Yb}\right]_{2}(\mu$-bipm $)$ is cationic $\mathrm{Cp}_{2}{ }_{2} \mathrm{Yb}(\text { bipy })^{+}$, where a neutral bipy ligand is coordinated to a trivalent, cationic $\mathrm{Cp}_{2}^{*} \mathrm{Yb}^{+}$center. ${ }^{36}$ The EPR spectrum of $\mathrm{Cp}^{*}{ }_{2} \mathrm{Yb}(\mathrm{bipy}) \mathrm{I}$ is shown in Figure 5. 
Although the symmetry is not truly axial since $g_{x}$ is not equal to $g_{y}, g_{z}$ is very different from $g_{x}$ and $g_{y}$, so the average of $g_{x}$ and $g_{y}$ was used to calculate $\gamma$. It should be noted that the ytterbocene complexes do not possess axial symmetry; therefore, the intermetallic axis cannot be assigned in this case, and was assumed to lie along the z-axis. While the intermetallic axis may be incorrectly assigned in these complexes, dipole-dipole coupling in these complexes is weak since the $\mathrm{Yb}$ centers are well separated. Therefore, incorrect assignment of the intermetallic axis has little effect on the analysis of exchange coupling in these systems As shown by the dashed line in Figure 6 , fitting the susceptibility of $\left[\mathrm{Cp}{ }_{2} \mathrm{Yb}\right]_{2}\left(\mu\right.$-bipm) using $\mathrm{Cp}_{2}{ }_{2} \mathrm{Yb}(\mathrm{bipy}) \mathrm{I}$ as the magnetically isolated analog is effective at low temperature and quite poor at high temperature. Two potential causes of this discrepancy are that $\mathrm{Cp}^{*}{ }_{2} \mathrm{Yb}(\mathrm{bipy}) \mathrm{I}$ is a poor analog or that the bridging bipm dianion has a low-lying triplet state that is populated above $90 \mathrm{~K}$. Based on the similarity of the $\mathrm{Yb}$ coordination environment in $\left[\mathrm{Cp}_{2}{ }_{2} \mathrm{Yb}\right]_{2}(\mu-\mathrm{bipm})$ and $\left[\mathrm{Cp}_{2}{ }_{2} \mathrm{Yb}(\mathrm{bipy})\right]^{+}\left[\mathrm{Cp}^{*}{ }_{2} \mathrm{YbCl}_{2}\right]^{-}$, which strongly suggests that $\mathrm{Cp}^{*}{ }_{2} \mathrm{Yb}($ bipy $) \mathrm{I}$ is a good analog, the possibility of a low-lying triplet state for the bipm dianion was examined.

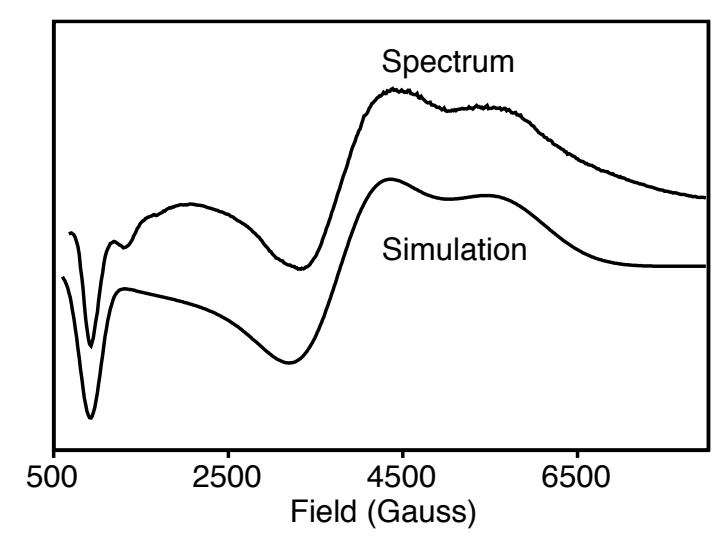

Figure 5. EPR spectrum of $\mathrm{Cp}^{*}{ }_{2} \mathrm{Yb}(\mathrm{bipy}) \mathrm{I}$ and simulation with $\mathrm{g}_{1}=7.050, \mathrm{~g}_{2}=1.731$, and $\mathrm{g}_{3}=1.165 ; \mathrm{g}_{1}$ is assigned as $\mathrm{g}_{\mathrm{z}}$, and the average of $\mathrm{g}_{2}$ and $\mathrm{g}_{3}$ was assigned as $\mathrm{g}_{\mathrm{x}}$. 


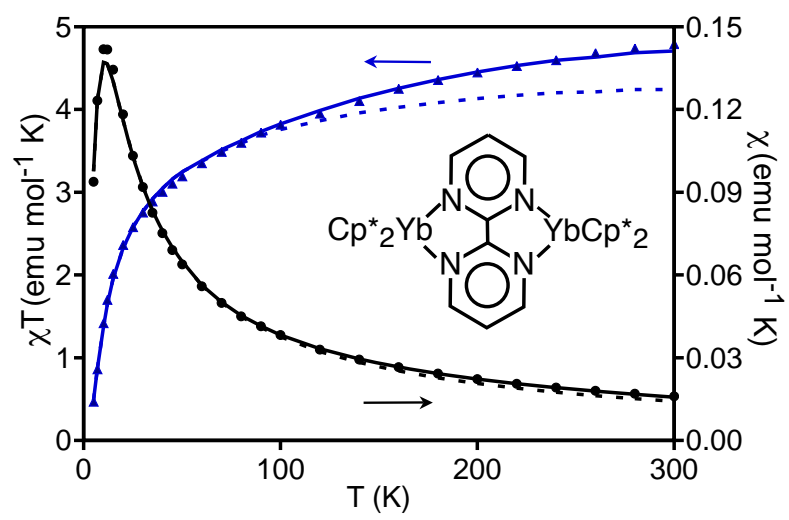

Figure 6. Magnetic susceptibility of $\left[\mathrm{Cp}_{2}{ }_{2} \mathrm{Yb}\right]_{2}(\mu$-bipm) (symbols) and fits (dashed lines) performed using eqs 15 and the anisotropy and susceptibility of the magnetically isolated analog, $\mathrm{Cp}_{2}{ }_{2} \mathrm{Yb}$ (bipy)I. The fit shown by the solid line includes a contribution from the triplet state of the bipm dianion. Susceptibility data are taken from ref. 35 .

The magnetic behavior of the bipm dianion has not been reported; however, neutral analogs, bis(verdazyl) diradicals, have been extensively studied. ${ }^{49-51}$ In particular, $\mathrm{Cu}(\mathrm{I})$ complexes of 1,1',5,5' -tetramethyl-6,6'-dioxobis(verdazyl) have singlet-triplet splittings ca. $\quad-200 \mathrm{~cm}^{-1}$; $^{49}$ therefore, it is plausible that the triplet state of bipyrimidine dianion could contribute to the susceptibility of $\left[\mathrm{Cp}_{2}{ }_{2} \mathrm{Yb}\right]_{2}(\mu$-bipyrimidine $)$. To examine this possibility, the contribution from a bipyrimidine dianion triplet state was included in the model, and the triplet-singlet splitting was allowed to vary. A much better fit was obtained (solid lines in Figure 6) with singlet-triplet splitting of $-207 \mathrm{~cm}^{-1}$. The close agreement with the singlet-triplet splitting of the $\mathrm{Cu}(\mathrm{I})$ bis(verdazyl) complexes is consistent with the postulate that the triplet state of the bridging bipyrimidine dianion contributes to the susceptibility above $\sim 90 \mathrm{~K}$. It is interesting to note that while the inclusion of the triplet state greatly improves the fit, it has little effect on the value of $\mathrm{J}$. 
In contrast to $\left[\mathrm{Cp}^{*}{ }_{2} \mathrm{Yb}\right]_{2}\left(\mu\right.$-bipm), the susceptibility of $\left[\mathrm{Cp}_{2}{ }_{2} \mathrm{Yb}\right]_{2}(\mu$-azb), does not show any evidence that the triplet state of the bridging ligand is thermally populated. The Yb centers in this complex are trivalent; therefore, as noted by Berg, et al., the bridging ligand in this complex is actually 1,2-diphenylhydrazide ${ }^{2-}$ rather than azobenzene. ${ }^{35}$ Therefore, a magnetically isolated analog would be a monodentate amide complex of $\mathrm{Cp}^{*}{ }_{2} \mathrm{Yb}$. A good candidate is $\left[\mathrm{Cp}_{2}{ }_{2} \mathrm{Yb}\right]_{2}(\mu-$ phz) where phz is phenazine. In this case, the bridging ligand is actually the dianion, 5,10 phenazide $^{2-}$, where the charge is localized on the nitrogen atoms, and the ytterbium centers are trivalent. The $\mathrm{Yb}-\mathrm{Yb}$ distance in $\left[\mathrm{Cp}_{2}^{*} \mathrm{Yb}\right]_{2}(\mu-\mathrm{azb})$ was determined from the analogous $\mathrm{Sm}$ complex reported by Evans, et al. ${ }^{52}$ No EPR spectrum has been reported for any $\mathrm{Cp}_{2}{ }_{2} \mathrm{Yb}$ amide complex, so the values of $\gamma$ and $\mathrm{g}_{\mathrm{z}}$ were determined from the EPR spectrum of $\mathrm{Cp}_{2}{ }_{2} \mathrm{Yb}(\mathrm{bipy}) \mathrm{I}$. The fit of the susceptibility of $\left[\mathrm{Cp}^{*}{ }_{2} \mathrm{Yb}\right]_{2}(\mu-\mathrm{azb})$ using $\left[\mathrm{Cp}_{2}{ }_{2} \mathrm{Yb}\right]_{2}(\mu-\mathrm{phz})$ as the magnetically isolated analog is shown in Figure 7. Unlike the previous case, the fit is good without having to include the contribution from the triplet state of the bridging ligand.

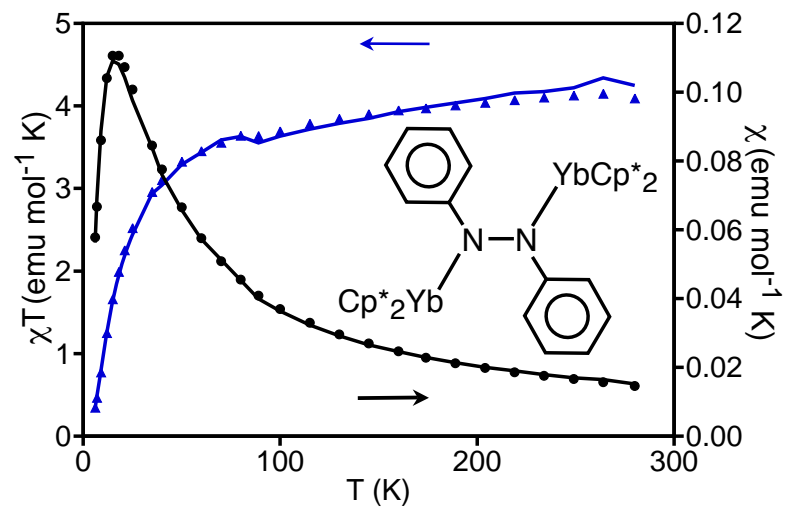


Figure 7. Magnetic susceptibility of $\left[\mathrm{Cp}_{2}{ }_{2} \mathrm{Yb}\right]_{2}(\mu$-azobenzene) (symbols) and fits (lines) performed using eq 18 and the anisotropy and susceptibility of the magnetically isolated analog, $\left[\mathrm{Cp}_{2}{ }_{2} \mathrm{Yb}\right]_{2}(\mu$-phenazine). Susceptibility data are taken from ref. 35 .

\section{Conclusion}

The quantitative diamagnetic substitution approach, which models the ground state exchange coupling of identical $f$-ions using the susceptibility of a magnetically isolated analog, allows exchange coupling between identical $f$-ions to be quantified without knowing the crystal field parameters. This method involves approximations that are valid when the crystal field is much stronger than the exchange coupling and when an appropriate magnetically isolated analog can be prepared. Although this method cannot provide a detailed description of the microscopic origin of exchange coupling (e.g. superexchange vs. spin-polarization) as it is based on a phenomenological HDVV Hamiltonian, this method does quantify the phenomenological coupling between the true spins of the $f$-electrons as well as the the effective spins. More importantly, this method provides a quantitative coupling constant, which can be compared to that predicted by a particular coupling mechanism, such as superexchange.

\section{Acknowledgements}

The authors wish to thank Richard Andersen, Corwin Booth, and Norman Edelstein for helpful discussions and are especially grateful to Richard Andersen for providing susceptibility data for the cerium, uranium, and decamethylytterbocene complexes. This work was supported by the Director, Office of Science, Office of Basic Energy Sciences, Chemical Sciences, Geosciences, 
and Biosciences Division, of the U.S. Department of Energy and by the Director, Office of Science, of the U.S. Department of Energy under Contract No. DE-AC02-05CH11231.

\section{Supplemental Material}

Figure showing the fit to the susceptibility of $\mathrm{COT}_{3} \mathrm{Ce}_{2}$ without a paramagnetic impurity contribution.

\section{References}

1. Benelli, C.; Gatteschi, D. Chem. Rev. 2002, 102, 2369-2387.

2. Rinehart, J. D.; Harris, T. D.; Kozimor, S. A.; Bartlett, B. M.; Long, J. R. Inorg. Chem. 2009, 48, 3382-3395.

3. Griffith, J. S. In Structure and Bonding; Hemmerich, P., Jorgensen, C. K., Neilands, J. B., Nyholm, R. S., Reinen, D., Williams, R. J. P., Eds.; Springer-Verlag: New York, 1972; Vol. 10, p 87-126.

4. $\quad$ Carlin, R. L. Magnetochemistry; Springer-Verlag: Berlin, 1986.

5. Kahn, O. Molecular Magnetism; Wiley-VCH: New York, 1993.

6. Booth, C. H.; Walter, M. D.; Daniel, M.; Lukens, W. W.; Andersen, R. A. Phys. Rev. Lett. 2005, 95, 4.

7. Walter, M. D.; Booth, C. H.; Lukens, W. W.; Andersen, R. A. Organometallics 2009, 28, 698-707.

8. $\quad$ Booth, C. H.; Walter, M. D.; Kazhdan, D.; Hu, Y. J.; Lukens, W. W.; Bauer, E. D.; Maron, L.; Eisenstein, O.; Andersen, R. A. J. Am. Chem. Soc. 2009, 131, 6480-6491.

9. $\quad$ Schelter, E. J.; Veauthier, J. M.; Thompson, J. D.; Scott, B. L.; John, K. D.; Morris, D. E.; Kiplinger, J. L. J. Am. Chem. Soc. 2006, 128, 2198-2199.

10. Ishikawa, N.; Iino, T.; Kaizu, Y. J. Phys. Chem. A 2002, 106, 9543-9550.

11. Ishikawa, N.; Iino, T.; Kaizu, Y. J. Am. Chem. Soc. 2002, 124, 11440-11447.

12. Ishikawa, N.; Sugita, M.; Ishikawa, T.; Koshihara, S.; Kaizu, Y. J. Am. Chem. Soc. 2003, $125,8694-8695$.

13. Ishikawa, N.; Satoshi, O.; Kaizu, Y. Angew. Chem.-Int. Edit. 2005, 44, 731-733.

14. Trojan, K. L.; Kendall, J. L.; Kepler, K. D.; Hatfield, W. E. Inorg. Chim. Acta 1992, 198, 795-803.

15. $\quad$ Sutter, J. P.; Kahn, M. L.; Kahn, O. Adv. Mater. 1999, 11, 863.

16. Kahn, M. L.; Sutter, J. P.; Golhen, S.; Guionneau, P.; Ouahab, L.; Kahn, O.; Chasseau, D. J. Am. Chem. Soc. 2000, 122, 3413-3421.

17. Schulz, H.; Reddmann, H.; Amberger, H. D.; Kanellakopulos, B.; Apostolidis, C.; Rebizant, J.; Edelstein, N. M. J. Organomet. Chem. 2001, 622, 19-32. 
18. Kahn, M. L.; Ballou, R.; Porcher, P.; Kahn, O.; Sutter, J. P. Chem.-Eur. J. 2002, 8, 525531.

19. Figuerola, A.; Diaz, C.; Ribas, J.; Tangoulis, V.; Sangregorio, C.; Gatteschi, D.; Maestro, M.; Mahia, J. Inorg. Chem. 2003, 42, 5274-5281.

20. Caneschi, A.; Dei, A.; Gatteschi, D.; Poussereau, S.; Sorace, L. Dalton Trans. 2004, 1048-1055.

21. Claiser, N.; Souhassou, M.; Lecomte, C.; Gillon, B.; Carbonera, C.; Caneschi, A.; Dei, A.; Gatteschi, D.; Bencini, A.; Pontillon, Y.; Lelievre-Berna, E. J. Phys. Chem. B 2005, 109, 2723-2732.

22. Bernot, K.; Bogani, L.; Caneschi, A.; Gatteschi, D.; Sessoli, R. J. Am. Chem. Soc. 2006, 128, 7947-7956.

23. Pointillart, F.; Bernot, K.; Sessoli, R.; Gatteschi, D. Chem.-Eur. J. 2007, 13, 1602-1609.

24. Przychodzen, P.; Pelka, R.; Lewinski, K.; Supel, J.; Rams, M.; Tomala, K.; Sieklucka, B. Inorg. Chem. 2007, 46, 8924-8938.

25. Yamaguchi, T.; Sunatsuki, Y.; Ishida, H.; Kojima, M.; Akashi, H.; Re, N.; Matsumoto, N.; Pochaba, A.; Mrozinski, J. Inorg. Chem. 2008, 47, 5736-5745.

26. Rinehart, J. D.; Bartlett, B. M.; Kozimor, S. A.; Long, J. R. Inorg. Chim. Acta 2008, 361, 3534-3538.

27. Sorace, L.; Sangregorio, C.; Figuerola, A.; Benelli, C.; Gatteschi, D. Chem.-Eur. J. 2009, $15,1377-1388$.

28. Abragam, A.; Bleaney, B. Electron Paramagnetic Resonance of Transition Ions; Clarendon Press: Oxford, 1970.

29. Tangoulis, V.; Estrader, M.; Figuerola, A.; Ribas, J.; Diaz, C. Chem. Phys. 2007, 336, 7482.

30. Santini, P.; Carretta, S.; Amoretti, G.; Caciuffo, R.; Magnani, N.; Lander, G. H. Rev. Mod. Phys. 2009, 81, 807-863.

31. Rosen, R. K.; Andersen, R. A.; Edelstein, N. M. J. Am. Chem. Soc. 1990, 112, 45884590 .

32. Spencer, L. P.; Schelter, E. J.; Yang, P.; Gdula, R. L.; Scott, B. L.; Thompson, J. D.; Kiplinger, J. L.; Batista, E. R.; Boncella, J. M. Angew. Chem.-Int. Edit. 2009, 48, 3795-3798.

33. Costes, J. P.; Dahan, F.; Dupuis, A.; Laurent, J. P. Chem.-Eur. J. 1998, 4, 1616-1620.

34. Kozimor, S. A.; Bartlett, B. M.; Rinehart, J. D.; Long, J. R. J. Am. Chem. Soc. 2007, 129, 10672.

35. Berg, D. J.; Boncella, J. M.; Andersen, R. A. Organometallics 2002, 21, 4622-4631.

36. Schultz, M.; Boncella, J. M.; Berg, D. J.; Tilley, T. D.; Andersen, R. A. Organometallics 2002, 21, 460-472.

37. Daul, C.; Schlapfer, C. W.; Mohos, B.; Ammeter, J.; Gamp, E. Comput. Phys. Commun. 1981, 21, 385-395.

38. Lines, M. E. J. Chem. Phys. 1971, 55, 2977.

39. Griffith, J. S. The Theory of Transition Metal Ions; Cambridge University Press: London, 1961.

40. Curely, J. Monatsh. Chem. 2005, 136, 1013-1036.

41. Elliott, R. J.; Thorpe, M. F. J. Appl. Phys. 1968, 39, 802-\&.

42. Palii, A. V.; Tsukerblat, B. S.; Clemente-Juan, J. M.; Coronado, E. Inorg. Chem. 2005, 44, 3984-3992.

43. Nakatsuka, S.; Osaki, K.; Uryu, N. Inorg. Chem. 1982, 21, 4332-4334. 
44. Elliott, R. J.; Stevens, K. W. H. Proc, Roy. Soc. A 1952, 215, 437-453.

45. Judd, B. R. Proc. Roy. Soc. A 1955, 232, 458-474.

46. Ishikawa, N.; Sugita, M.; Ishikawa, T.; Koshihara, S.; Kaizu, Y. J. Phys. Chem. B 2004, 108, 11265-11271.

47. Chandrasekaran, R. Acta Cryst. B 1969, B 25, 369-\&.

48. Brennan, J. G.; Andersen, R. A. J. Am. Chem. Soc. 1985, 107, 514-516.

49. Brook, D. J. R.; Lynch, V.; Conklin, B.; Fox, M. A. J. Am. Chem. Soc. 1997, 119, 51555162.

50. $\quad$ Fico, R. M.; Hay, M. F.; Reese, S.; Hammond, S.; Lambert, E.; Fox, M. A. J. Org. Chem. 1999, 64, 9386-9392.

51. Koivisto, B. D.; Hicks, R. G. Coord. Chem. Rev. 2005, 249, 2612-2630.

52. $\quad$ Evans, W. J.; Drummond, D. K.; Chamberlain, L. R.; Doedens, R. J.; Bott, S. G.; Zhang, H. M.; Atwood, J. L. J. Am. Chem. Soc. 1988, 110, 4983-4994. 
TOC Synopsis:

Exchange coupling in complexes containing pairs of lanthanide or actinide ions may be quantified using the magnetic susceptibility and EPR spectrum of a complex with a similar crystal field but no exchange coupling, which may prepared by the diamagnetic substitution method.

TOC Graphic

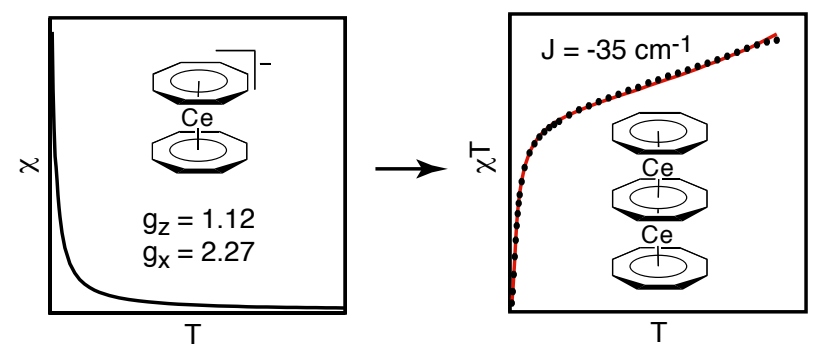

